\title{
ROBUST INTERACTIVE VIDEO TRANSMISSION IN MC-CDMA CELLULAR NETWORKS OVER SLOW FADING CHANNELS
}

\author{
Mario R. Hueda and Carmen E. Rodríguez
}

\begin{abstract}
Reliable interactive video transmission in CDMA cellular networks is difficult because residual channel errors (after error correction) cannot be completely climinated and the available bandwidth is scarce. To improve the quality of the decoded video, this paper introduces a novel architecture for low bit rate transmission in multicode DS-CDMA (MC-CDMA) cellular systems. Unlike previous protection schemes, the new architecture not only improves video quality, but it also achieves an efficient use of the available resources in the network. As an application of this concept, we propose a robust scheme for reliable video communication from wired to mobile users in slow fading environments. This scenario has been shown to be the most critical owing to long block error bursts present in the forward link of CDMA systems where closed-loop power control is not used (such as IS-95B), and the inability of error resilience approaches incorporated in wired video codecs to guarantee an acceptable video quality in wireless transmissions. Our approach consists of two main ingredients: (i) a novel closed-loop error control technique to reduce packet loss, delays, and interference, and (ii) a new scheme to stop error propagation in the decoded sequence originating from residual channel errors. Based both on theoretical analysis and numerical results, we show that our approach achieves important improvements in the quality of the decoded video. Furthermore, we show that the new architecture outperforms previous error protection schemes with low additional complexity
\end{abstract}

Keywords: multicode, CDMA, wireless video transmission, ARQ, error resilience.

Resumo - Uma transmissão confiável de vídeo interativo em redes celulares CDMA é difícil de se obter devido ao fato de os erros residuais do canal (após a correção de erros) não poderem ser eliminados por completo, e devido à escassez da largura de faixa de frequências disponível. Com o propósito de melhorar a qualidade do vídeo decodificado, este artigo apresenta uma nova arquitetura para a transmissão em baixa taxa de bit em sistemas celulares multicódigo DS-CDMA (MC-CDMA). Diferente dos esquemas de proteção existentes, a nova arquitetura não apenas melhora a qualidade de vídeo, mas também proporciona um uso eficiente dos recursos disponíveis na rede. Como uma aplicação desse conceito, propomos um esquema robusto para comunicação confiâvel de vídeo tanto para os usuários móveis quanto para os fixos em ambientes com desvanecimento lento. É sabido que esse é o cenário mais crítico

The authors are with the Digital Communications Research Laboratory, National University of Cordoba, Cordoba, Argentina. Email: mhueda @com.uncor.edu. Review coordinated by Renato Baldini Filho (Area Editor). Manuscript received May/08/2003, reviewed Sept/04/2003, accepted Oct/02/2003. devido à presença de longos surtos de erros no enlace direto de sistemas CDMA, onde o controle de potencia de malha fechada não é utilizado (como no IS-95B), e devido ao fato de as abordagens de resiliência a erros incorporadas aos "codecs" usados para transmissão por fio não serem capazes de garantir uma qualidade de vídeo aceitável em sistemas de transmissão sem fio. Nossa ahordagem consiste em dois importantes ingredientes: (i) uma nova técnica de controle de erros em malha fechada para reduzir a perda de pacotes, os atrasos e a interferência, e (ii) um novo esquema para eliminar na sequiência decodificada a propagação de erro que se origina a partir dos erros residuais do canal. Baseados na análise teórica e nos resultados numéricos, mostramos que a abordagem proposta melhora significativamente a qualidade do vídeo decodificado. Além disso, mostramos que a nova arquitetura apresenta melhor desempenho em comparação aos esquemas de proteção de erro existentes com um pequeno acréscimo de complexidade.

Palavras-chave - multicódigo, CDMA, transmissão sem fio de vídeo, ARQ, resiliência a erros.

\section{INTRODUCTION}

Multicode direct sequence code division multiple access (MC-CDMA) has been adopted by several standards to support variable data rates [1]-[3]. The viability of high speed service offerings in MC-CDMA systems depends on the number of data users that the system can support while providing adequate capacity for voice users [4]-[6]. These services may require dedicated allocation of bandwidth for the duration of the session or they may be provided bandwidth on a burst-by-burst basis. For example, it has been shown in [6] that the 64 kilobits per second (kbps) service cannot be offered as a continuous bit rate service in IS-95B, and bandwidth has to be allocated to users on a burst basis. However, dedicated bandwidth service offerings may be viable at lower rates (e.g., up to 4 multicode channels per user [6], or $38.4 \mathrm{kbps}$ for rate set 1 of IS-95B). Because real time applications such as video require a dedicated bandwidth, low data rates are unavoidable in transmission over MC-CDMA systems if reasonable capacity is to be reserved for voice users (e.g. $<32$ kbps for IS-9.5B).

Transmission of real time video over wireless networks has received considerable attention in recent litcrature. In particular, there has been interest in the transmission of H.263/H.26.3+ coded video sequences [7]-[9] over mobile networks. However, H.263/H.263 + coded video data are extremely sensitive to channel errors, so additional protection schemes must be incorporated [10]. Three types of schemes have been used to improve video quality in transmission over error-prone channels: error concealment, 
error correction, and error resilience. Error concealment techniques refer to those error post-processing methods where the video decoder, recognizing that an uncorrectable error has occurred, seeks to hide or minimize the glitch as observed by the viewer, so that a more visually pleasing rendition of the decoded video can be obtained [11]. Error correction methods are used to decrease the packet losses owing to transmission over error-prone channels, while error resilience approaches attempt to curtail the spread and severity of the damage originating from transmission errors. The video quality achieved at the decoder in transmission over error-prone channels depends critically on both error correction and error resilience techniques. The ability of the latter to improve video quality in MC-CDMA wireless transmission al low rates requires further considerations. For example, increasing the frequency of refresh with an intra-coded frame (i.e., a coded video frame that can be decoded by itself) to stop error propagation is not a proper solution because this frame has low compression efficiency and significant additional bandwidth is required to reduce time delays. This not only affects the overall video quality and system capacity (it increases interference) [12], but it also requires elaborate bandwidth allocation algorithms. On the other hand, adjusting the percentage of intra-coded macroblocks (I-MBs) [1.3] is a solution that cannot be achieved without suffering high distortion in the case of video transmission at low bit rates. A possible solution to improve video quality in MC-CDMA transmissions consists in combining an error resilience method with an error correction scheme [14].

To achieve robust low rate video transmission in $\mathrm{MC}$ CDMA systems, we adopt a protection architecture centralized at the base station. As we shall show later, this new concept not only improves the video quality decoded at a mobile station, but it also achieves an efficient use of the available resources in an MC-CDMA network. Although our approach is general, we focus here on the problem of providing reliable interactive video communication from users in a wired network (e.g., an H.324 based user) [15] to mobile users (e.g., an IS-95B mobile station) in slow fading environments. This scenario has been shown to be the most critical owing to (i) long block error bursts present in the forward link of CDMA systems where closed-loop power control is not used [1]. and (ii) the inability of error resilience approaches incorporated in wired video codecs to guarantee an acceptable video quality in wireless transmissions [10], [16]. The architecture proposed in this work consists of $(i)$ a novel closed-loop error control technique to reduce packet loss, called the non-selective variable bandwidth retransmission scheme (NSVBR), and (ii) a new scheme to stop error propagation in the decoded sequence originating from residual channel errors, which is called the centralized video quality recovery (CVQR) scheme. NSVBR is a new retransmission strategy designed not only to reduce delays and packet loss, but also interference and complexity of the code (bandwidth) allocation algorithm (e.g., it can be easily incorporated into existing MC-CDMA systems such as IS-95B). On the other hand, in CVQR a base station improves the video quality achieved at a mobile station by transmitting a video frame especially coded to stop error propagation in the decoded sequence. Based both on numerical results and theoretical analysis, we show that the proposed technique achieves important improvements in the quality of the decoded video. Furthermore, we show that the new architecture outperforms previous error protection schemes with low additional complexity. The rest of this paper is organized as follows. A new error protection scheme for reliable wireless video transmission over $\mathrm{MC}$ CDMA cellular networks is discussed in Section 2. NSVBR and CVQR are addressed in Section 3 and 4, respectively. The performance of the new architecture is evaluated in Section 5, while concluding remarks are given in Section 6.

\section{ERROR PROTECTION FOR ROBUST VIDEO TRANSMISSION IN MC-CDMA}

\subsection{PRELIMINARY}

Without loss of generality, in this work we analyzt H.26.3 based video transmission over an IS-95B system. H.263 is a compression standard developed to transmit video using the telephone network at data rates less than 64 kbps [7]. The picture resolution at low bit-rates is often QCIF (Quarter Common Intermediate Format, 176×144 pixels). At QCIF resolution, each picture is divided into $11 \times 9$ macroblocks (MBs), which comprise $16 \times 16$ luminance samples, and two corresponding $8 \times 8$ blocks of chrominance samples. A fixed number of successive MBs is usually grouped into a group of blocks (GOB). We assume that synchronization words are inserted at the beginning of each macroblock row of a GOB. This is exploited by the error concealment technique employed at the decoder, which discards corrupted GOBs and replaces the corresponding image content with data from the previously decoded frame. A typical H.263 video stream is composed of intra-coded frames ( $I$-frames) and predictive-coded frames ( $P$-frames). An $l$-frame is an independently coded video frame that can be decoded by itself. A $P$-frame is composed of changes in the current image of the video stream relative to the last $I$-frame encoded. An $H .263$ video stream is usually an $I$-frame followed by many $P$-frames, with an 1 -frame re-introduced to restore image quality in case of transmission errors.

In MC-CDMA, high speed is provided through code aggregation. For example. up to eight multicode channels (MCCs) may be assigned in 1S-95B (one fundamental code channel (FCC) and seven supplemental code channels (SCCs)), providing a maximum bit rate of $115.2 \mathrm{kbps}$ [1]. As a result of limited system capacity and the power consumption constraints of the mobile stations, interactive video services over MC-CDMA cellular systems are almost universally limited to low data rates [6]. Owing to the scarce bandwidth available, video transmission based on $P$ frames cannot be avoided. As a consequence of the facts that (i) long block-error bursts occur at the input of the video decoder in DS-CDMA transmission over slow fading channels, and (ii) coded video data are extremely sensitive to channel errors (because transmission of $P$-frames is assumed), the error propagation problem in the decoder video is severe [16], [17]. Therefore, protection schemes fo robust wireless video transmission must be incorporated. 


\subsection{ERROR PROTECTION SCHEMES FOR CDMA}

A traditional protection process for robust wireless video transmission takes place between end-users. This approach, however, may be not suitable for interference limited CDMA wireless cellular networks. To justify this claim, consider a correction scheme based on a retransmission protocol used between two mobile stations (MSs), $u_{I}$ and $u_{2}$. Note that information (and retransmission) must go through reverse and forward links of two different CDMA cells. Furthermore, MSs may have different characteristics (e.g., mobility, bandwidth, etc.), and their cells might have different properties (e.g., power control schemes, load, etc.).

Suppose now that the signal received by user $u_{1}$ is in a deep fade and the channel variation is slow. In this situation, user $u_{2}$ retransmits lost packets with low efficiency due to the long duration of the signal fade experienced by user $u_{1}$. Therefore, the resources of $u_{2}$ are used inefficiently (e.g., the error rate is not decreased by retransmissions), so the additional interference generated by retransmissions over other users from its cell is not exploited.

To overcome this problem inherent in traditional protection schemes, in this work we adopt an architecture centralized at the base station (BS) where each mobile user together with its BS is considered as an independent link. In this way it is possible not only to improve the users" performance and video quality, but also to achieve an efficient use of the available resources in the network. Following the previous example and assuming a good quality of the link $u_{2} \leftrightarrow \mathrm{BS}$, a closed-loop error control technique between $u_{1}$ and its $\mathrm{BS}$ could reduce the interference originating from the retransmission process over the other users in the cell of $u_{2}$. Furthermore, it can be useful to achieve a more robust video transmission between a wired user and an MS. This problem has received considerable attention in recent work [14], [18], [19]. In this case, a protection scheme centralized at the BS would allow a better utilization of the scarce resources available in wired links, such as bandwidth. In this paper we focus on robust interactive video transmission from a wired user (e.g., an $\mathrm{H} .324$ based user) to an MC-CDMA user (e.g., an IS-95B user) in slow fading environments. In the following we introduce a new error correction scheme based on a retransmission protocol with variable bandwidth.

\subsection{A NOVEL VARIABLE BANDWIDTH RETRANSMISSION SCHEME FOR ROBUST VIDEO TRANSMISSION IN MC- CDMA OVER SLOW FADING CHANNELS}

In MC-CDMA transmissions over slow fading channels, the use of forward error correction codes (FECs) [20] is limited owing not only to the long bit error burst characteristics [21], but also to the bandwidth restriction of MCCDMA systems. Therefore, feedback-based error control techniques need to be considered [22]. In particular, retransmission schemes such as automatic repeat request (ARQ) reduce packet loss. This allows a more efficient use of error resilience techniques to further improve the error resilience of video transmission [14], [23]. Since real time video services are delay-bounded and CDMA systems are interference limited. retransmission protocols must be designed in a way that minimizes both time delays and interference.

Numerous retransmission schemes for wireless video transmission have been proposed in previous work [21], [24]-[29]. For example, Hsu et. al. [25] introduced rate control algorithms combined with ARQ for robust video transmission over wireless channels, while Tosun and Feng proposed in [24] a retransmission scheme combined with $\mathrm{BCH}$ error correction to increase the quality of video services in limited bandwidth DS-CDMA systems. However, these approaches have not been devised for MCCDMA systems (e.g., IS-95B) where the bandwidth restriction needs to be considered. Furthermore, retransmission schemes designed to reduce not only packet loss and delays, but also interference have not been reported so far. This factor is critical to improve the system capacity (i.e., the number of users) because CDMA systems are interference limited. Therefore, retransmission protocols aimed at robust wireless video transmission in practical MC-CDMA systems must be designed in a way that reduces $(a)$ video distortion, $(b)$ packet loss, $(c)$ time delays, (d) interference, as well (e) complexity of bandwidth allocation algorithms.

Video transmission over MC-CDMA has received considerable attention in recent literature (e.g., [12], [30][32], [44]). In particular, Fitzek et. al. reported in [32] several link-layers transmission strategies for multicode CDMA systems. Basically, these methods do not transmit new information when the channel state is bad (probing time), and store in buffers the incoming information. Then, as soon as the channel state improves, extra multicode channels in burst-mode are used to transmit all buffered information. These strategies, however, have not been designed for interactive video services. In addition, the time delay required by the burst admission in existing $\mathrm{MC}$ CDMA systems (e.g., IS-95B) may be not tolerable for interactive applications [4], [33]. Furthermore, the strategies described in [32] are not efficient in slow fading environments where long bursts of blocks with errors occur, because (i) the probing time is large so the delay introduced is unacceptable; (ii) the number of multicodes required to transmit the information when the channel state improves is large (this increases both interference to other users in the cell and the peak to average ratio (PAR) at the transmitter).

To overcome these limitations, in this work we introduce the variable bandwidth retransmission scheme (VBR), a novel ARQ strategy designed to reduce packet loss, time delays, interference, and complexity in transmission of realtime services at low data rates over MC-CDMA networks. In VBR, retransmission takes place between the MS and its BS. Because interactive video requires a bounded delay, only one retransmission is allowed in VBR. Moreover, depending on the round trip delay and the number of MCCs, only a limited number of packets can be retransmitted. To allow block retransmission with low time delay, user bandwidth is increased. Although this can be accomplished by varying the number of multicode channels assigned to a given user as in [32], VBR adjusts the data rate of FCC. The latter method is available in several CDMA 
standards such as IS-95B, where it is typically used to exploit the voice activity factor [3]. Thus, VBR not only avoids underutilizing scarce bandwidth, but it also significantly reduces the complexity of the bandwidth allocation algorithm, allowing its easy integration into existing CDMA systems such as IS-95B. Perhaps even more important, VBR can be used to reduce packet loss in transmission of interactive video, where the serious delays restrictions (e.g., smaller than $200 \mathrm{~ms}$ ) do not allow to adopt strategies such as those reported by Fitzek et al. in [32].

This work is oriented towards slow fading channels. This motivates the use of a non-selective VBR (NSVBR) where consecutive data blocks are retransmitted when a negative acknowledge (NACK) is received. Note that this approach reduces complexity at the mobile station at the expense of efficiency (its performance is worse than that of selective schemes because some data blocks may be retransmitted even if they had been correctly received in the first transmission). We have verified, however, that this degradation is not important when the round trip delay is small (recall that NSVBR takes place between the MS and its BS) and the channel varies slowly [34]. In this way, NSVBR achieves a good tradeoff between efficiency and complexity, as we show in the next section.

\section{ANALYSIS OF NSVBR}

Let $N, M$ and $R_{t o t}$ denote the round trip delay in slots, the number of MCCs assigned to a given user, and the total user bit rate for video, respectively. In "normal conditions" (i.e., no errors), the FCC works at a data rate $R_{F C C_{n o r}}$, less than its maximal $R_{F C C_{m a x}}$. This transmission mode will be denoted by $M_{1}$. The value of $R_{F C C_{n o r}}$ depends on link quality (e.g., the system load), and is determined at the beginning of the communication (the optimal value of $R_{F C C_{n o r}}$ will be considered in subsection 3.4). For example, if the average error rate of the link is moderately high, a low value of $R_{F C C_{n o r}}$ is selected (i.e., the video bandwidth (quality) is reduced), thus a "high" bandwidth ( $R_{F C C_{\text {max }}}-R_{F C C_{\text {nor }}}$ ) is reserved for retransmissions. When the receiver (e.g., a mobile station) detects only one of the $M$ received blocks with error, an NAK is sent to the transmitter (e.g., the base station). This approach simplifies implementation at the expense of efficiency. However, we have verified that the degradation is not important since the block error processes among MCCs are highly correlated [34]. Then, when the transmitter receives an NAK, the data rate of FCC is increased to $R_{F C C_{m a x}}$ and the retransmission (only one) of MCC blocks begins. This transmission mode will be denoted by $M_{2}$. Note that not all the $N M$ blocks are sent again since the bandwidth assigned for retransmission is limited. Only a fraction of the lost information is retransmitted. For example, consider $N=6$ and the following transmission rates $R_{F C C_{n o r}}=7.2 \mathrm{kbps}$, $R_{F C C_{\text {mat }}}=14.4 \mathrm{kbps}\left(R_{t o t}=R_{F C C_{n t r}}+R_{S C C}=21.6 \mathrm{kbps}\right)$. Then, when an NAK is received, only

$$
\delta=\frac{R_{F C C_{m a t}}-R_{F C C}}{R_{t o t}}=0.33
$$

or $33 \%$ of the $N M=12$ blocks can be retransmitted. When the retransmission process (or $M_{2}$ ) finishes, the system returns to the "normal" mode (i.e., $M_{\mathrm{I}}$ ).

Note that the ability of NSVBR to reduce packet loss depends both on the round trip delay and the number of MCC. However, since low data rates are considered here, NSVBR provides a good tradeoff between efficiency and complexity, as we shall show later.

\subsection{A MARKOV MODEL FOR THE BLOCK- ERROR PROCESS IN MC-CDMA}

We present a Markov model for block-error processes in MC-CDMA systems, which will be used here for theoretical analysis [35]. Let $\beta_{i, n}$ be a binary process such that

$\beta_{i, n}=1$ if the data block $i$ at the channel decoder output of the $n^{t h}$ multicode is in error, and 0 otherwise. We define another process $\boldsymbol{\Psi}_{i}$ called super-block as

$$
\Psi_{i}=\left(\beta_{i, 0}, \beta_{i, 1}, \ldots, \beta_{i, M-1}\right) .
$$

Based on an information theoretic argument, we showed in [35] that the superblock process $\boldsymbol{\Psi}_{i}$ is well approximated by a $2^{M}$ - state Markov process, with transition matrix $\boldsymbol{\Pi}_{M}(x)=\boldsymbol{\Pi}_{M}(1)^{x}$, where

$$
\begin{aligned}
\boldsymbol{\Pi}_{M}(x) & =\left[\begin{array}{ccc}
m_{0,0}(x) & \cdots & m_{0,2^{M}-1}(x) \\
\vdots & \vdots & \vdots \\
m_{2^{M}-1,0}(x) & \cdots & m_{2^{M}-1,2^{M}-1}(x)
\end{array}\right], \\
\boldsymbol{\Pi}_{M}(1) & =\left[\begin{array}{ccc}
m_{0,0} & \cdots & m_{0,2^{M}-1} \\
\vdots & \vdots & \vdots \\
m_{2^{M}-1,0} & \cdots & m_{2^{M}-1,2^{M}-1}
\end{array}\right],
\end{aligned}
$$

with $m_{u, v}(x) u, v=0,1,2, \ldots, 2^{M}-1$, representing the probability of $\boldsymbol{\Psi}_{i}=\mathbf{v}$ in slot $i$ given that the super-block in slot $i-x$ was $\Psi_{i-x}=\mathbf{u}$ ( $\mathbf{n}$ denotes the $M$-dimensional binary vector representation of the number $n$ ). The Markov model describing this process is called the "super-block Markov" model" (SBMM). An efficient method to estimate the elements $m_{u, u^{\prime}}$ is presented in [35].

\subsection{PERFORMANCE OF NSVBR}

Based on the SBMM, in the following we investigate the performance of NSVBR. In particular, we focus our analysis on the capability of NSVBR to reduce packet loss and interference. 
We define a main block as the concatenation of $M$ packet blocks, with each packet block corresponding to each one of the $M$ multicode channels. In NSVBR, a main block is considered to be in error if at least one multicode block is in error. Thus we can assume that the main block error process is a first-order Markov process described by the matrix $\boldsymbol{\Pi}_{M B}(x)=\boldsymbol{\Pi}_{M B}(1)^{x}$, with

$$
\begin{gathered}
\boldsymbol{\Pi}_{M B}(x)=\left[\begin{array}{ll}
p_{M B}(x) & q_{M B}(x) \\
r_{M B}(x) & s_{M B}(x)
\end{array}\right], \\
\boldsymbol{\Pi}_{M B}(1)=\left[\begin{array}{ll}
p_{M B} & q_{M B} \\
r_{M B} & s_{M B}
\end{array}\right],
\end{gathered}
$$

where $q_{M B}=1-p_{M B}, s_{M B}=1-r_{M B}$,

$$
\begin{gathered}
p_{M B}=\operatorname{Pr}\left\{\boldsymbol{\Psi}_{i}=\mathbf{0} \mid \boldsymbol{\Psi}_{i-1}=\mathbf{0}\right\}, \\
\sum_{M B} \operatorname{Pr}\left\{\boldsymbol{\Psi}_{i}=\mathbf{0} \mid \boldsymbol{\Psi}_{i-1}=\mathbf{x}\right\} \operatorname{Pr}\left\{\boldsymbol{\Psi}_{i-1}=\mathbf{x}\right\} \\
1-\operatorname{Pr} \overline{\{}\left\{\boldsymbol{\Psi}_{i}=\mathbf{0}\right\}
\end{gathered}
$$

$\Xi$ is the set of the possible values of $\boldsymbol{\Psi}_{i}$ (e.g., $\Xi=\{(00),(01),(10),(11)\}$ for $M=2)$. Model parameters $\operatorname{Pr}\left\{\boldsymbol{\Psi}_{i}=\mathbf{X} \mid \boldsymbol{\Psi}_{i-1}=\mathbf{Y}\right\}$ and $\operatorname{Pr}\left\{\boldsymbol{\Psi}_{i}=\mathbf{X}\right\}, \quad \mathbf{X}, \mathbf{Y} \in \Xi$, are obtained from (14) and (15) in [35], respectively. Since the process is stationary, it is not difficult to show that

$$
r_{M B}=\frac{\operatorname{Pr}\left\{\Psi_{i}=0\right\}\left(1-\operatorname{Pr}\left\{\Psi_{i}=0 \mid \Psi_{i-1}=0\right\}\right)}{1-\operatorname{Pr}\left\{\Psi_{i}=0\right\}}
$$

Using (5) and (7), the elements of the transition matrix $\Pi_{M B}(1)$ can be easily determined.

\section{a) Residual Packet Error Rate}

Because there are two operation modes in NSVBR $\left(M_{1}\right.$ and $M_{2}$ ), we define the following transition matrices:

$$
\begin{aligned}
\mathbf{\Pi}_{M B-M_{1}}(x) & =\left[\begin{array}{ll}
p_{M B-M_{1}}(x) & q_{M B-M_{1}}(x) \\
r_{M B-M_{1}}(x) & s_{M B-M_{1}}(x)
\end{array}\right], \\
\boldsymbol{\Pi}_{M B-M_{1}}(1) & =\left[\begin{array}{ll}
p_{M B-M_{1}} & q_{M B-M_{1}} \\
r_{M B-M_{1}} & s_{M B-M_{1}}
\end{array}\right] ; \\
\boldsymbol{\Pi}_{M B-M_{2}}(x) & =\left[\begin{array}{ll}
p_{M B-M_{2}}(x) & q_{M B-M_{2}}(x) \\
r_{M B-M_{2}}(x) & s_{M B-M_{2}}(x)
\end{array}\right], \\
\mathbf{\Pi}_{M B-M_{2}}(1) & =\left[\begin{array}{ll}
p_{M B-M_{2}} & q_{M B-M_{2}} \\
r_{M B-M_{2}} & s_{M B-M_{2}}
\end{array}\right] .
\end{aligned}
$$

Using (8), the marginal error probability of the main block in state $M_{1}, \varepsilon_{M B-M_{1}}$, can be expressed as [34]

$$
\varepsilon_{M B-M_{1}}=-\frac{q_{M B-M_{1}}}{q_{M B-M_{1}}+r_{M B-M_{1}}}
$$

Note that (10) represents the packet error probability for the system without retransmissions. NSVBR improves system efficiency reducing $\varepsilon_{M B-M_{1}}$ through limited retransmissions. Therefore, an expression for the residual packet error rate (RPER) is required to evaluate NSVBR performance.

Let $N_{r} \in\{0,1, \ldots,\lfloor\delta N\}$ be the number of retransmitted main blocks in NSVBR, with $\delta$ defined by (1) (symbol L.] denotes the integer part). Notice that the maximal number of retransmitted main blocks in NSVBR,

$$
N_{r_{\max }}=\lfloor\delta N\rfloor=\delta N
$$

depends on $N, R_{t o r} \cdot R_{F C C_{n o r}}$, and $R_{F C C_{\text {max }}}\left(N_{r_{\text {max }}}=2\right.$ for the numerical example presented previously (see (1))). Note also that (i) every $N_{r}$ consecutive retransmitted blocks, there are $N-N_{r}$ consecutive main blocks not retransmitted; (ii) retransmission of $N_{r}$ main blocks requires $N_{s}=\left\lceil N_{r} / \delta\right\rceil$ time slots in mode $\mathcal{M}_{2}$, with $\lceil\cdot\rceil$ denoting the nearest integer larger than the argument. For example, $N_{s}=N_{r_{\max }} / \delta=N$ is the maximal number of time slots in the mode $M_{2}$ required to retransmit $N_{r_{\text {max }}}$ main blocks.

Based on these observations and (8)-(10), the RPER can be well approximated at low values of $\varepsilon_{M B-M_{1}}$ by (no feedback error is assumed') [34]

$$
\begin{aligned}
R P E R \approx \varepsilon_{M B-M}, M_{1} & \left\{1-r_{M B-M_{1}}\left(N_{r}\right) .\right. \\
& \left.\left(p_{M B-M_{1}}\right)^{N-N_{r}-1}\left(p_{M B-M_{2}}\right)^{N_{s}}\right\}
\end{aligned}
$$

The second term in the expression enclosed in brackets represents the reduction of the block error probability owing to the single retransmission. Moreover, from (12) we verify that $R P E R \rightarrow \varepsilon_{M B-M}$ when no main block is retransmitted $\left(N_{r}=0 \Rightarrow r_{M B-M_{1}}\left(N_{r}\right)=0\right.$ ) or the round trip delay is very large; in a limit case we obtain

$$
\lim _{N \rightarrow \infty} R P E R=\varepsilon_{M B-M_{1}}
$$

This occurs because as $N$ grows, not only $N_{r}$ increases, but the number of non-retransmitted main blocks, $N-N_{r}$, also increases. The latter effect gives rise to an increased number of lost packets, therefore the overall performance tends to that of an unprotected system.

\section{b) Activity Factor of FCC}

In NSVBR, the data rate of the FCC is increased to achieve the retransmission process with low delay. This originates additional interference that affects system capacity. In order to analyze the interference effects in NSVBR, we use the activity factor of the FCC given by

$$
F=E\{\alpha\}
$$

\footnotetext{
' Similar to [22], we assume that acknowledgments (ACKs) and NACKs are received error-free after a relatively large round trip delay between base and mobile stations (e.g., $100 \mathrm{~ms}$ ).
} 
where $E\{\}$ denotes the expectation operator and $\alpha$ is the random variable defined as

$$
\alpha=\left\{\begin{array}{ccc}
R_{F C C_{n o r}} / R_{F C C_{\text {max }}} \text { for } & M_{1} \\
1 & \text { for } & M_{2}
\end{array}\right.
$$

The activity factor (14) can be rewritten as

$$
\begin{aligned}
& F=\operatorname{Pr}\left\{\mathcal{M}_{1}\right\} R_{F C C_{n a r}}^{R_{\text {max }}}+\operatorname{Pr}\left\{\mathcal{M}_{2}\right\}= \\
& =1-\operatorname{Pr}\left\{\mathcal{M}_{1}\right\} \frac{R_{F C C_{\text {max }}}-R_{F C C_{\text {nor }}}}{R_{F C C_{\max }}},
\end{aligned}
$$

where $\operatorname{Pr}\left\{\mathcal{M}_{1}\right\}$ and $\operatorname{Pr}\left\{S_{2}\right\}=1-\operatorname{Pr}\left\{S_{1}\right\}$ are the probabilities of modes $M_{1}$ and $M_{2}$, respectively. In the special case of $N_{r}=N_{r_{m a x}}$ (i.e., $N_{s}=N$ ), we note that the probability of $M_{1}$ is equivalent to the reciprocal of the average number of retransmissions (including the first transmission), required for a packet to be successfully accepted by the receiver in a conventional $G B N$ scheme. Therefore, $\operatorname{Pr}\left\{\mathcal{M}_{1}\right\}$ can be obtained from the throughput of GBN as follows [35]

$$
\operatorname{Pr}\left\{M_{1}\right\} \approx \frac{r_{M B-M_{2}}(N)}{r_{M B-M_{2}}(N)+N q_{M B-M_{1}}}
$$

This result can be easily generalized for any value of $N_{r}$ :

$$
\operatorname{Pr}\left\{M_{1}\right\} \approx \frac{r_{M B-M_{2}}(N)+\left(N-N_{s}\right) q_{M B-M_{1}}}{r_{M B-M_{2}}(N)+N q_{M B-M_{1}}} .
$$

Based on (16) and (17), we conclude that:

- $\quad F_{\min }=R_{F C C_{n o r}} / R_{F C C_{\text {max }}}$ corresponds to the case of error free transmission $\left(q_{M B}-M_{1}=0\right)$ or no retransmission $\left(N_{r}=N_{s}=0\right)$.

- At high block error rates $\left(N q_{M B-M_{1}}>r_{M B-M_{2}}(N)\right)$, $\operatorname{Pr}\left\{\Lambda M_{1}\right\} \approx\left(N-N_{s}\right) / N$ and $F \rightarrow F_{\max }$ with $F_{\text {max }}=F_{\text {min }}+\left(1-F_{\text {min }}\right) N_{s} / N$. For example, the maximal activity factor results in $F_{\max }=1$ for $N_{s}=N$, which indicates that blocks retransmissions occur during all the transmission time.

- At low block error rates $\left(N q_{M B-M_{1}} \ll r_{M B-M_{2}}(N)\right)$, $\operatorname{Pr}\left\{\mathcal{M}_{1}\right\} \rightarrow 1$ and $F \rightarrow F_{\text {min }}$. The latter result implies that the extra interference owing to the retransmission process is low, which is highly desirable in CDMA systems.

\subsection{NUMERICAL RESULTS AND DISCUSSION}

We use the parameters, interleaver/deinterleaver, and convolutional code of the downlink of the IS-95B standard [1]. Furthermore, we use soft-decision decoding of the rate $3 / 4$, constraint length 9 , convolutional code (i.e., rate set 2 of IS-95B). We set the number of RAKE fingers, $L$, to four ( $L=4$ ) [36]. We assume that RAKE fingers have equal power. The block rate is $1 / T_{b}=50$ block/s. A 16 -bit cyclic redundancy code (CRC) is used for block error detection. Carrier frequency is $1800 \mathrm{MHz}$ and the fading rate is $f_{m} T_{b}=0.04$, where $f_{m}$ is the maximal Doppler frequency. Since the rate variation of the channel is small, we assume ideal coherent demodulation at the RAKE receiver [36]. Throughout this work, we assume Rayleigh fading and adopt the Gaussian approximation for the interference-plusnoise component [35], [37]. The Rayleigh channel is simulated using Jakes' model [38]. We set the number of multicodes to two $(M=2)$. FCC and an SCC are used to transmit video (we assume that voice is carried by an additional SCC). The rate of SCC is $14.4 \mathrm{kbps}$. Since the transmission rate of the fundamental code channel can be adjusted in practical systems, we consider three rates of FCC: 14.4. 7.2, and $3.6 \mathrm{kbps}$, which will be denoted by $R S O, R S 1$, and $R S 2$, respectively. In all of the cases, 2.4 kbps are used for overhead (i.e., packet headers + CRC + tail block). Therefore, the net video bit rates are 26.4, 19.2 and $15.6 \mathrm{kbps}$ for $R S O, R S I$, and $R S 2$, respectively. Table 1 shows a summary of the transmission rates considered in this work.

Figure 1 shows the RPER vs. average signal-tointerference plus noise ratio (SINR) per bit for $R S 1$ $(\delta=0.33)$ and $R S 2 \quad(\delta=0.6)^{2}$. Several values of $N$ are considered. Moreover, we assume that no feedback errors occur [22] and $N_{r}=\lfloor\delta N\rfloor$. Analytical (12) and entire system simulation results show that significant reduction of packet loss is obtained with the proposed retransmission scheme. The excellent agreement between results obtained from theory and simulation can also be verified. From Figure 1 we notice that higher gains are achieved for $R S 2$ since the retransmission bandwidth is increased. Moreover, note that the performance of NSVBR improves as $N$ increases from 3 to 9 , as a result of the increased number of retransmitted main blocks, $N_{r}$. We have verified, however, that the gain of NSVBR decreases at higher values of $N$ according to (13).

On the other hand, the round trip delay must be bounded for an acceptable quality of the reproduced video (e.g., a time delay around of $100 \mathrm{~ms}$ has been suggested for the case of video transmission at low data rates [21]).

\begin{tabular}{|c|c|c|c|c|}
\hline $\begin{array}{c}\text { Trans. } \\
\text { Rate }\end{array}$ & $\begin{array}{c}\text { Bit Rate } \\
\text { of FCC } \\
\text { (kbps) }\end{array}$ & $\begin{array}{c}\text { Total Bit } \\
\text { Rate } \\
\text { (FCC+SCC } \\
\text { - kbps) }\end{array}$ & $\begin{array}{c}\text { Overhead } \\
\text { Bit Rate } \\
\text { (kbps) }\end{array}$ & $\begin{array}{c}\text { Video Net } \\
\text { Bit Rate } \\
\text { (kbps) }\end{array}$ \\
\hline$R S 0$ & 14.4 & 28.8 & 2.4 & 26.4 \\
\hline$R S 1$ & 7.2 & 21.6 & 2.4 & 19.2 \\
\hline$R S 2$ & 3.6 & 18.0 & 2.4 & 15.6 \\
\hline
\end{tabular}

Table 1. Rates used in this paper. Number of multicodes $M$ $=2$. Bit rate of $\mathrm{SCC}=14.4 \mathrm{kbps}$.

In NSVBR, $N$ is around 4-6 slots since the retransmission process is carried out between the base and the mobile stations. Therefore, from the above we conclude that NSVBR improves the transmission efficiency introducing an acceptable time delay for the video services considered in this work.

\footnotetext{
${ }^{2}$ Based on Table 1, (1), and (16), note that $\delta=0$ and $F=1$ for rate
} RSO. 

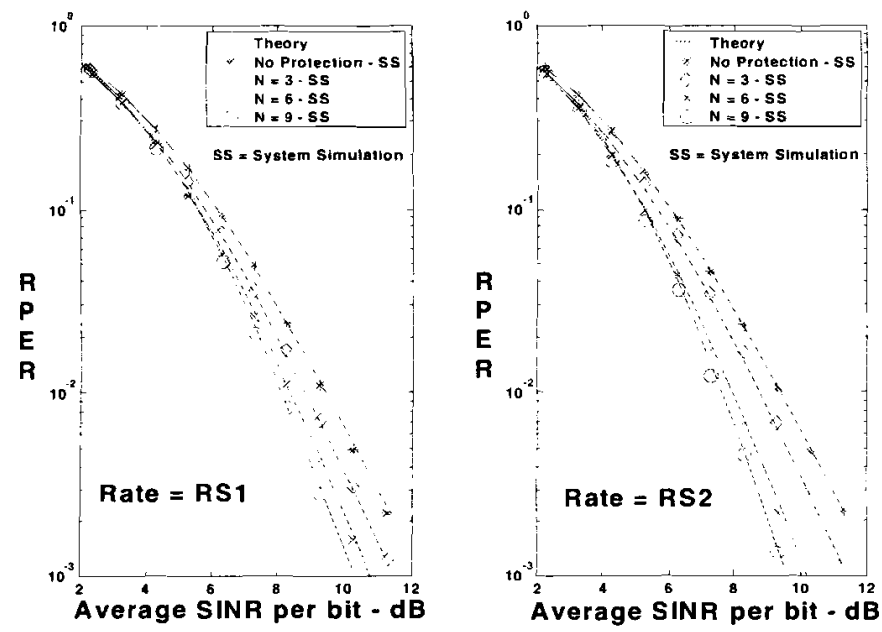

Figure 1. Performance of NSVBR. RPER vs. average SINR per bit.
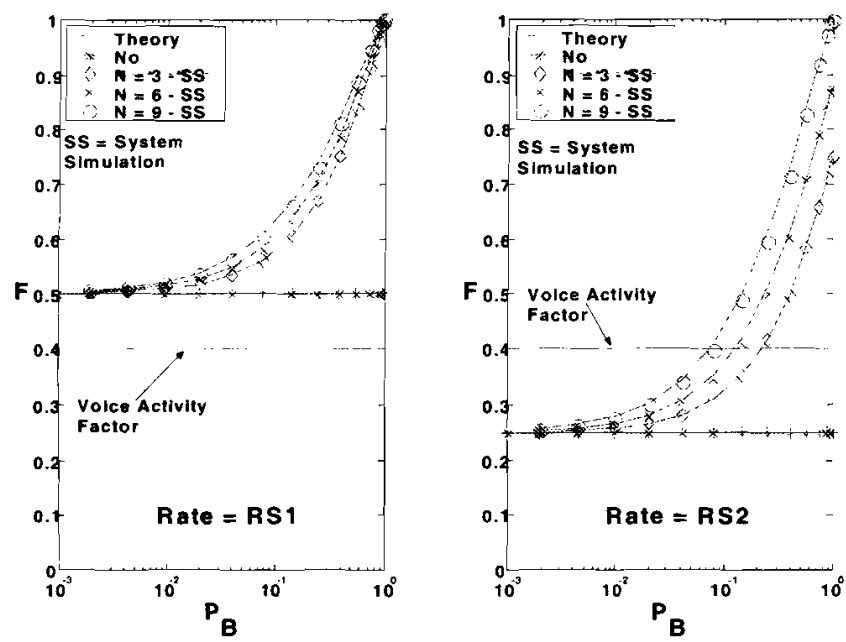

Figure 2. Performance of NSVBR. Activity Factor of FCC vs. average block error of the SCC.

The activity factors of the FCC, $F$, versus the average block error of the SCC, $F$, for $R S 1\left(F_{\min }=0.5\right)$ and $R S 2$ $\left(F_{\min }=0.25\right)$ are analyzed in Figure 2 . We observe an excellent agreement between theoretical (16)-(17) and entire system simulation results. We also notice that the activity factor grows as $N$ increases, as a result of the decrease of $\operatorname{Pr}\left\{M_{1}\right\}$ owing to the increased number of retransmitted main blocks, $N_{r}$. Note that for practical values of $P_{B} \quad(<0.1)$ and $N(\leq 6), F$ is around $0.5-0.65$ for $R S 1$ and $0.25-0.38$ for $R S 2$. On the other hand, it is well known that the voice activity factor is about 0.4 . Therefore, when FCC is used for data retransmission instead of voice, we conclude that improvements of the system capacity are obtained for $R S 2$ while an overall degradation of the capacity occurs for $R S 1$. In this case, however, an important gain of our scheme is observed when it is compared with an equivalent protocol with fixed bandwidth for retransmission (i.e., $F=1)^{3}$.

\footnotetext{
${ }^{3}$ Furthermore, voice activity can also be exploited in NSVBR. For this, in silent periods we can reduce the FCC bit rate and transmit 308
}

From Figs. 1 and 2 it can be noted that packet loss and interference are minimized using transmission rate $R S 2$. This shows the advantages of using a variable bandwidth retransmission scheme that adjusts the data rate of FCC. In this way it is possible to minimize not only packet loss, delays, and interference, but also the complexity of the bandwidth allocation algorithm. This enables its easy integration into existing CDMA systems (variable data rate for the FCC is available in most CDMA standards, such as IS-95B).

\subsection{PERFORMANCE OF VIDEO TRANSMISSION USING NSVBR}

Note that, although RS2 minimizes packet loss and interference, the quality of the coded video at this rate will be the worst since the net video bit rate in $R S 2$ is the lowest. Therefore, evaluation of video performance is required to determine first the overall video quality for different transmission rates, and then the optimal value for $R_{F C C_{n o r}}$. This is addressed in this subsection. We focus on peaksignal to noise ratio (PSNR), which has been widely adopted as a distortion measure. The PSNR of the video reproduced at the receiver in transmission over error-prone channels (after the error concealment stage) is given by

$$
\operatorname{PSNR}(t)=10 \log _{10}\left[\frac{255^{2}}{D_{S C}(t)+D_{C H}(t)}\right],
$$

where $D_{S C}(t)$ is the video source coding distortion and $D_{C H}(t)$ is the distortion owing to transmission errors [13] (only the PSNR of the luminance is computed in this paper). The loss of picture quality $\triangle P S N R(t)$ is also used as a measure of the video degradation due to channel errors. It is defined as [23]

$$
\Delta P S N R(t)=10 \log _{10}\left[\frac{D_{S C}(t)}{D_{S C}(t)+D_{C H}(t)}\right] .
$$

To perform our simulation experiments, we use the University of British Columbia's H.263+ Reference codec. The rate control method discussed in TMN-8 [39] is used in our simulations. All of the results represent around 160 pictures of test sequences having QCIF resolution, coded at 6 frames per second (fps). The error concealment technique described in subsection 2.1 is used. Although numerous simulations have been performed, only a few results are discussed in this paper because of space constraints. Specifically, we analyze the loss of picture quality for the test sequences Mother\&Daughter and Foreman. These sequences are selected because of their different characteristic in motion and spatial detail [13]. We use the general simulation conditions adopted in the previous subsection. Three channel states are analyzed in this paper: "Bad", "Good", and "Very Good", which have an SCC average block error of $P_{B}=0.08,0.041$, and 0.02 ,

the video packets (or/and retransmission) by using the voice SCC. In a similar way, additional improvements can be achieved by decreasing the bit rate for voice (available in practical systems), and increasing thus the bandwidth for retransmission. 
respectively. These values correspond approximately to 19 , 11 , and 6 data users per cell (with one code per user) in a typical multicell transmission environment [14].
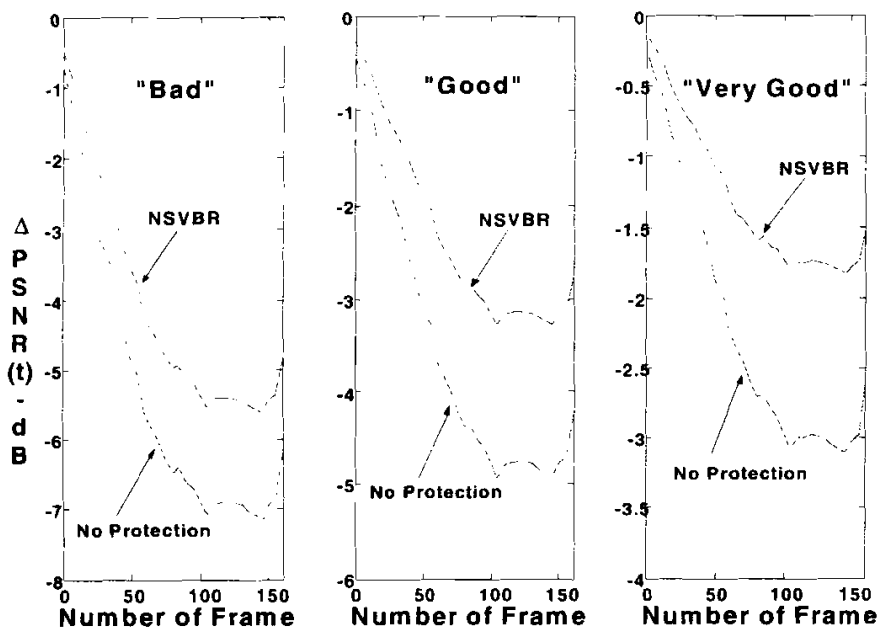

Figure 3. Results for QCIF Mother\&Daughter sequence in IS-95B using NSVBR. Channel states: "Bad", "Good", and "Very Good". Round trip delay $N=6$. Rate $=R S I$.
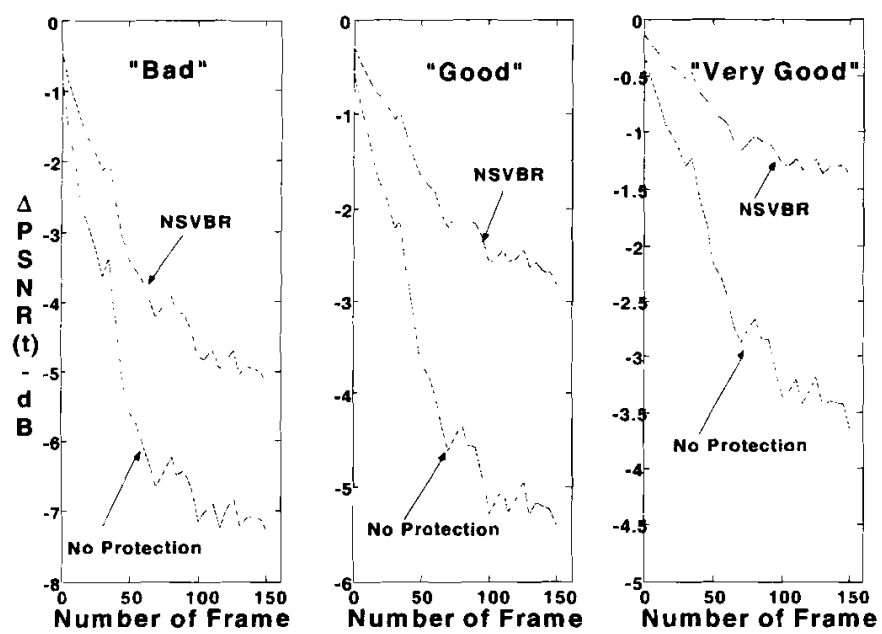

Figure 4. Results for QCIF Foreman sequence in IS-95B using NSVBR. Channel states: "Bad" , "Good", and "Very Good". Round trip delay $N=6$. Rate $=R S 2$.

The losses of picture quality $\triangle P S N R(t)$ for the video test sequences Mother\&Daughter (rate RSI) and Foreman (rate $R S 2$ ) with $N=6$ are presented in Figs. 3 and 4 , respectively. We present results from entire system simulation, where the coded sequences were transmitted 300 times using different starting points in the fading simulator. The average over all the runs is presented.

Compared with an unprotected system, a significant gain can be observed for both test sequences. This gain depends on the residual block error rate (12) and the amount of motion presem in the sequence.

Figure 5 shows the average $P S N R(t)$ versus the normal transmission rate of FCC $\left(R_{F C C_{n o r}}\right)$ obtained from entire system simulations for NSVBR with $N=6$. We also include curves for transmission with no retransmission and no error. Compared with error-free transmissions, we see that video quality degradation for both systems (i.e., with/without NSVBR) decreases as $R_{F C C_{n o r}}$ does. Note also that the gains achieved by NSVBR grow as the rate of FCC decreases because the capability of NSVBR to reduce packet loss improves as a result of the bigger bandwidth for retransmission. In particular, the biggest gains are obtained for $R S 2\left(R_{F C C_{n o r}}=3.6 \mathrm{kbps}\right.$ and $\left.\delta=0.6\right)$, while no gain is verified for $R S O \quad\left(R_{F C C}=14.4 \mathrm{kbps} \rightarrow \delta=0 \rightarrow\right.$ no retransmissions). It is interesting to observe that the average video quality for $R S I$ ( $R_{F C C_{m o r}}=7.2 \mathrm{kbps}$ ) in transmission over the channel state "Good" with NSVBR, is approximately the same as that one obtained in transmissions over the channel state "Very Good' with no retransmission.
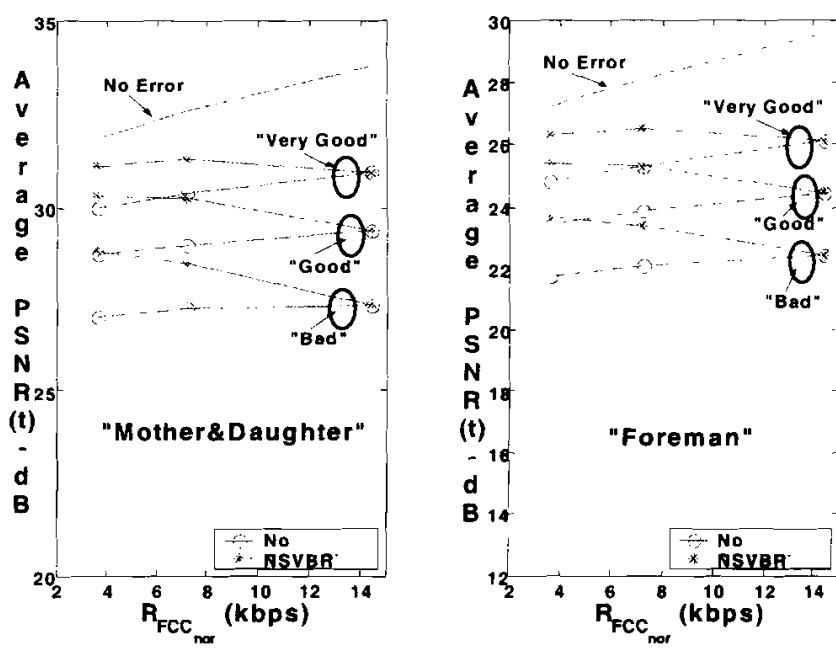

Figure 5. Average PSNR vs. transmission rate of FCC using NSVBR for several channel states. Round trip delay $N=6$.

From Figure 5 we also verify that $R S I$ is the optimum rate for the channel state "Veny Good" ( $F \approx 0.51)$, while $R S 2$ is the best selection for the channel states "Good" ( $F \approx 0.29$ ) and "Bad" ( $F \approx 0.38$ ). Note, however, that the video quality at $R S 2$ for the state "Very Good" $(F \approx 0.26)$ is slightly lower than the optimal. Therefore, we conclude that NSVBR with RS2 achieves the best tradeoff between video quality and interference.

In this situation, the average data activity factor of FCC is around 0.3 . Since the voice activity factor is around 0.4 , we verify that improvements of both video quality and system capacity are obtained with our scheme. Furthermore, note that these important improvements are achieved with reduced additional complexity and without modifying the H.263 video encoder.

\section{NEW ERROR RESILIENCE METHOD}

Although NSVBR significantly improves the forward link performance in slow fading environments, it is clear that residual transmission errors cannot be avoided in a mobile radio channel. Furthermore, it is well known that a given loss of the PSNR caused by these errors is usually more serious than the same loss caused by higher compression since the resulting artifacts are concentrated in a part of the picture and hence more annoying. Therefore, additional schemes to stop the spatio-temporal error prop- 
agation have to be considered. Our NSVBR can be efficiently combined with error resilience techniques to further improve robustness of coded video transmission in $\mathrm{MC}$ CDMA.

Numerous error resilience techniques have been proposed in the literature to improve video quality in transmissions over error-prone channels [13], [23], [40], [41]. For example, Rhee and Joshi introduced in [41] an interesting error recovery scheme called RESCU, which alleviates error propagation in transmission of interactive video over the Internet. This scheme, however, is not suitable for low bit rate video transmission over MCCDMA systems in slow fading channels because it requires a significant bit overhead to keep an acceptable video quality, and the recovery process can fail due to the presence of long block error bursts [41].

Among the error resilience techniques used in transmission over mobile channels, the error tracking (ET) approach has been shown to achieve a good tradeoff between video quality and complexity [23]. ET utilizes intra-coded MBs ( $I$-MBs) refresh to stop interframe error propagation. Using a feedback channel, the time and position of an error are reported to the transmitter. With this information, the location and extent of propagated errors are reconstructed at the encoder. In practical situations, quality degradation is experienced because:

- to maintain constant bit rate, only a fraction of MBs corresponding to severely affected image regions are coded in $l$-mode;

- video source coding distortion increases as a result of the higher number of $I$-MBs;

- packet losses between the last frame for which acknowledgment was received and the current frame, are not considered by the transmitter.

Better results than ET can be achieved, for example, with the error resilience technique described by $\mathrm{R}$. Zhang et. al. in [40]. However, this approach still uses 1 -MB refresh and requires modifications of $\mathrm{H} .263$ video encoders, estimate of the packet loss rate, and heavy numerical computation (it works at the pixel level). All these inconveniences are overcome by the centralized video quality recovery (CVQR) scheme, which is described in the following.

\subsection{DESCRIPTION OF CVQR}

Based on the feedback channel used by the retransmission protocol (i.e., NSVBR), the base station is able to reconstruct the video decoded at the mobile station. Towards this end, the BS analyzes the ACKs/NACKs provided by the retransmission protocol. Then, taking into account the error concealment technique used by the MS, the decoded video can be reconstructed at the BS. On the other hand, the original video transmitted from the wired user (WU) can be easily decoded at the BS. Then, since the original and reproduced video frames are known at the BS. it is possible to obtain a measure of the video degradation level at the MS. When this value is larger than a certain threshold, the BS transmits a new frame called correction frame (CF), which is coded based on the difference between the original and reproduced video frames. To reduce delays,

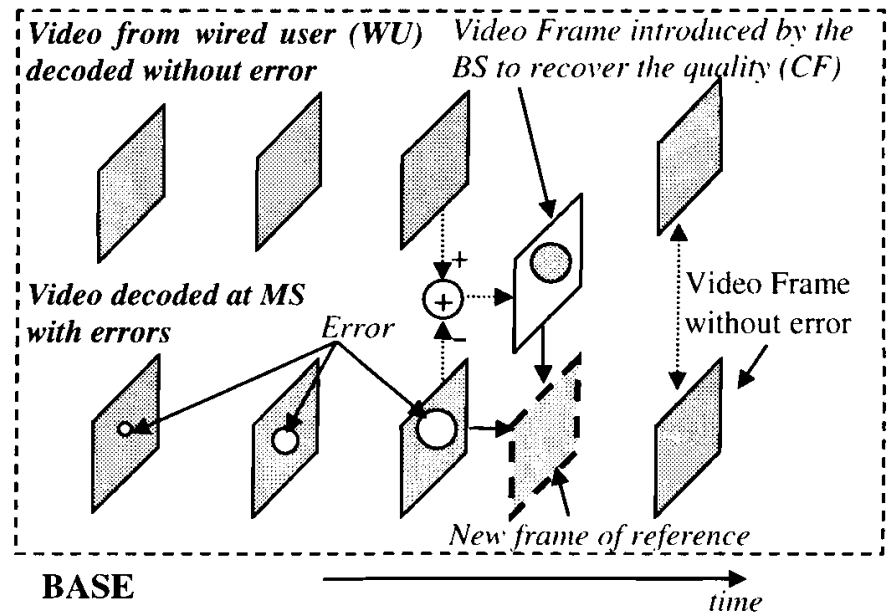

Figure 6. New CVQR scheme to recover video quality

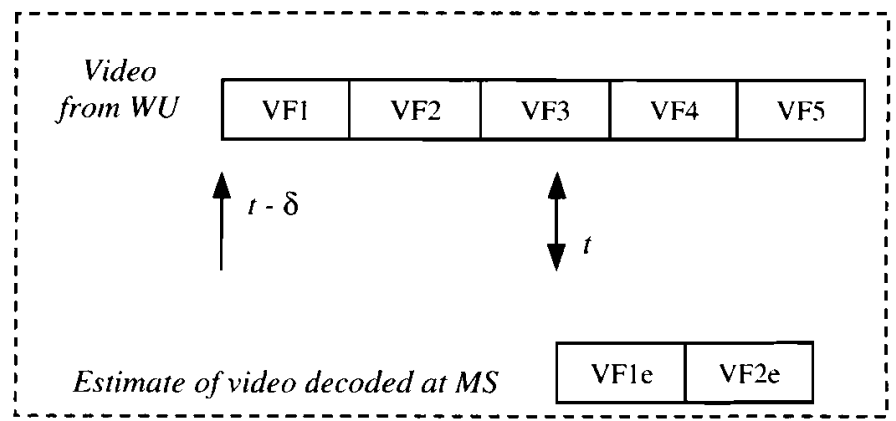

\section{BASE STATION}

Figure 7. Reconstruction at the BS of video received from WU and decoded at MS.

the transmission of the CF corresponding to instant $t-1$ and the actual video frame (instant $t$ ) is achieved increasing the user's bandwidth. At the MS, the CF is decoded first and subsequently the actual video frame is decoded. Assuming that there is no information loss in the coding process of $\mathrm{CF}$, the actual frame will be decoded with the same reference utilized by the WU's video encoder; this way the error propagation will be stopped (see Figure 6).

\subsection{IMPLEMENTATION OF CVQR IN SLOW FADING ENVIRONMENTS}

Because of the use of NSVBR, the BS is able to reconstruct at instant $t$ the video frame (VF) decoded by the MS at instant $t-\delta$ (Figure 7). Since the round trip delay $(N)$ and the frame rate are small (e.g., $N \leq 6$ slots and $6 \mathrm{fps}$, respectively), it can be verified that $\delta$ is around 2-3 video frames (the slot duration is $20 \mathrm{~ms}$ in IS-95B). On the other hand, since the channel varies slowly, the MS can estimate the channel state with good accuracy (e.g., using the pilot signal), and report it to the BS. Based on this information, the video quality recovery process is achieved as follows (see Figure 8). If the BS detects at $t_{0}$ that $(i)$ the $\mathrm{BS} \rightarrow \mathrm{MS}$ link is good (i.e., a strong signal level at the MS), and (ii) the degradation of the decoded video is significant, the CVQR process is started. BS knows at instant $t_{2}$ how the 
data packets corresponding to VFl (and the previous ones) were received at the MS after the retransmission process. In addition, the BS can determine if there were errors in the original transmission of VF2 (i.e., with no retransmission).

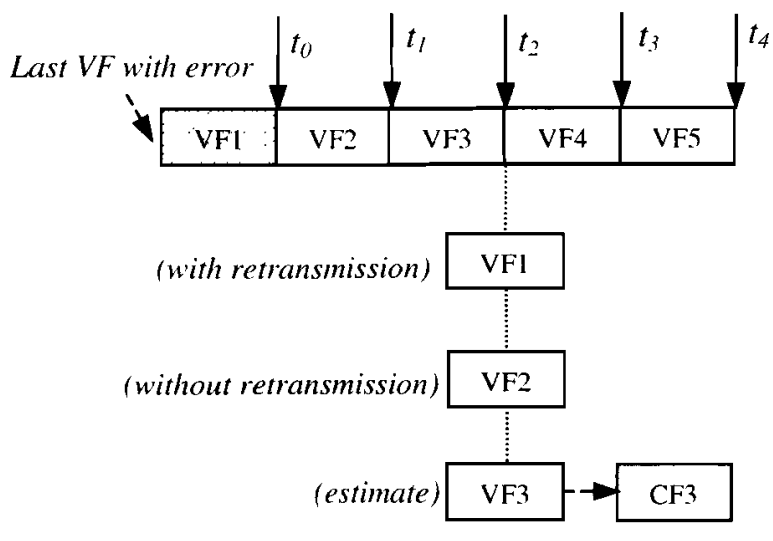

Figure 8. Estimates of correction frame (CF).

In this case, it is expected that the data blocks with information of VF2 are received with no errors because of the good quality of the BS $\rightarrow$ MS link (if FV2 is received with error, CVQR will try again at $t_{3}$ ). Thus, the BS can reconstruct exactly the VFs decoded at the MS corresponding to VF1 (and the previous ones) and VF2 (the reconstructed video frames will be denoted by $\mathrm{VFX}_{\mathrm{e}}$ ). Furthermore, a good estimate of $V F 3_{\mathrm{e}}$ can be obtained at instant $t_{2}$. Towards this end, we assume that the data blocks with information of VF3 will be transmitted with no errors. This is based on the facts that the signal level at the MS is good and the channel varies slowly. This way, the BS can estimate at instant $t_{2}$ the correction frame (CF) required to stop error propagation (i.e., CF3 $=\mathrm{VF} 3-\mathrm{VF} 3_{\mathrm{e}}$ ). Then CF3 is encoded and the BS increases the user's bandwidth to transmit both $\mathrm{CF} 3$ and VF4 with low delay. It is important to realize that part of the required bandwidth can be obtained from the retransmission channel used by NSVBR. This is possible since the bandwidth for retransmission will be available due to good link conditions (in this case, the ideal probability of data loss is zero). At the MS, the video decoder will process CF3 first and then VF4 (note that the delay introduced is negligible). Assuming that the coding of CF3 is ideal (i.e., there is no information loss), VF4 will be decoded with the same reference used by the WU's video encoder, and thus, the error propagation will be stopped. When the CVQR process finishes, the system returns to the normal operation (e.g., at instant $t_{3}$ ).

In practical situations, the $\mathrm{CF}$ is coded with loss, therefore a residual degradation will be observed in the decoded sequence. However, this degradation is not important for video sequences with low/medium motion as a result of: $(i)$ the low recovery time delay, $T_{C V Q R}$ (CVQR takes place between an MS and its BS), (ii) the use at MS of an error concealment method, (iii) and the fact that CF is coded from the difference between two frames (like a $P$ frame). In sequences with heavy motion, the transmission of CF requires extra bandwidth to achieve an acceptable degradation level. When this extra bandwidth is not available, the CVQR process can be repeated several times to gradually reduce video distortion. Figure 9 shows the PSNR obtained with CVQR for video test sequences Mother\&Daughter (1-stage CVQR) and Foreman (1 and 2stage CVQR). The bandwidth required to transmit the correction frames is provided by the retransmission channel (i.e., $10.8 \mathrm{kbps}$ for $R S 2$ ). From Figure 9 it can be verified that the residual video degradation is negligible. Note also that owing to the heavy motion contained in sequence Foreman, a 2-stage CVQR process is required to achieve an acceptable video quality without requiring extra bandwidth.

\subsection{DISCUSSION}

- Unlike ET, CVQR takes into account how all VFs were received by the mobile station. For VFs previous to VF3, the BS has the information required to calculate exactly the VFs reproduced by the MS (we consider that no feedback errors occur). On the other hand, VF3. is estimated assuming that data blocks of VF3 will be received without errors due to the "good" state of the channel. CF3 is obtained from the difference between VF3 and VF3 ${ }_{\mathrm{e}}$. Taking into account the presence of the error concealment technique used by the video decoder and the low recovery time delay (e.g., $T_{C V Q R} \approx 4-5$ VFs), it can be inferred that video degradation will not be significant (in other words, the mean number of bits required to encode CF3 will be small). This way, the bandwidth requirements are not important and can be satisfied by the retransmission channel. Note that in this situation the performance degradation of NSVBR (due to the use of the retransmission channel) will be minimal, since the recovery process is achieved when the channel state is "good" (i.e., there are no lost packets).

- CVQR does not use intra-coded MBs so the original video quality from $\mathrm{WU}$ is not degraded.

- Video quality recovery is successful if VF3 and CF3 are received correctly. On the other hand, we have verified that the average length of the sequence of video frames without errors in transmissions over slow fading channels is higher than 5 (this study is not included in this paper). Therefore, it can be inferred that the probability that VF3 and CF3 are correctly received is high since the CVQR takes place when the channel state is good. If VF3 or CF3 are received with error, it is just required to start the CVQR process at $t_{3}$ or $t_{4}$, respectively (see Figure 8 ).

- The additional complexity required at the MS is minimal. Only a modest increase of memory is necessary to store several coded video frames. In addition, a simple scheme to control and order data blocks is required. Note that, with suitable control logic at the MS, it is not necessary to modify existing video decoders.

- BS can reconstruct video frames reproduced at the MS using ACKs/NACKs provided by a feedback error control scheme. Therefore, it is important to protect this information in order to assure its correct reception at the BS. Nevertheless, since the WU transmits an Iframe refresh frequently (e.g., each 132 coded frames), it is possible to prevent propagation of long error 
events of indeterminate duration caused by incorrect ACKs/NACKs. Furthermore, this problem can be overcome by means of transcoding at the BS [18], and using CVQR to reduce the video distortion caused by the transcoding process.
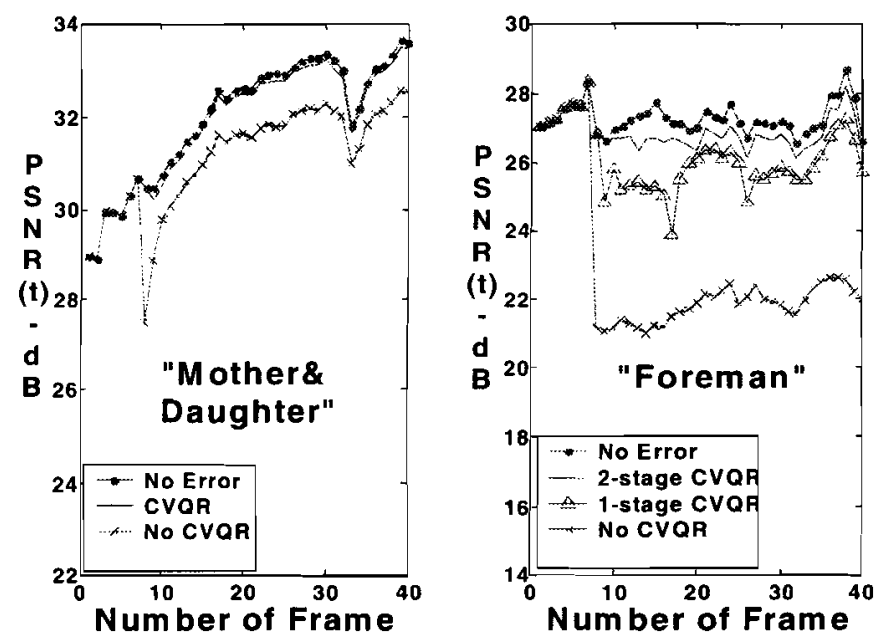

Figure 9. Effectiveness of $\mathrm{CF}$ to recover video quality. $P_{B}=$ 0.041 .

\section{PERFORMANCE OF THE PROPOSED ARCHITECTURE}

In this section we investigate performance of the architecture composed by NSVBR+CVQR. In particular, we compare the quality of the decoded video achieved by our approach with that obtained from an enhanced error tracking approach (E-ET) and an ideal error resilience technique (I-ER) [42]. E-ET is similar to the technique presented in [23]. However, unlike [23], E-ET assumes that the video source coding distortion $D_{S C}(t)$ is not affected by $I-\mathrm{MBs}$ refresh. On the other hand, I-ER is a theoretical approach that assumes that errors in a particular video frame do not propagate. This way, note that I-ER achieves the best performance of an error resilience technique for a given transmission environment (e.g., block error probability).

\subsection{EVALUATION OF VIDEO DEGRADATION}

To determine the performance of the protection scheme proposed in this paper, in the following we evaluate the video degradation of $P$-frames owing to channel errors, $D_{C H}(t)$ (see (18)). Towards this end, we use the model for $D_{C H}(t)$ described in the Appendix. This model provides an efficient alternative to computer simulation in the analysis and design of these relatively complex systems. Unlike previous work [13], our model can be used to accurately analyze low bit rate transmission of video sequences even in the presence of rapid motion and fast changes of the motion pattern [42].
From (A-6), and assuming that

- the recovery time delay $T_{C V Q R}$ is constant (i.e., it does not depend on the video degradation level at the MS or link quality),

- the error signal at $t=0$ is successfully canceled at $t=T_{C V Q R}$, and

- the error concealment technique discards a corrupted region of the image and replaces it with data from the previously decoded frame.

it can be verified that the video degradation owing to channel errors achieved by NSVBR+CVQR is given by [14]

$$
D_{C H}(t)=\sum_{k=0}^{\infty} D^{C V Q R}\left(t, k T_{C V Q R}\right)
$$

with

$$
\begin{aligned}
D^{C V Q R}(t, \tau)= & P_{B . R} G \cdot\left\{[\Gamma(t) u(t-\tau)] \oplus \frac{1}{1+\theta .(t-\tau)}\right\} \\
& \cdot\left[u(t-\tau)-u\left(t-\tau-T_{C V Q R}\right)\right]
\end{aligned}
$$

where $u(t)$ is the unit step function and $\oplus$ denotes convolution. $P_{B, R}$ is the residual block error probability (which depends on several factors such as channel code, number of RAKE fingers, round trip delay, etc.), and it is given by (12) for NSVBR. The leakage $\boldsymbol{\theta}$ describes the efficiency of explicit and/or implicit (e.g., due to sub-pel motion compensation, overlapped block transmission) loop filtering to remove the introduced error. Its value depends on the strength of the loop filtering as well as on the shape of the power spectral density of the introduced error. $G \Gamma(t)$ represents the sensitivity of the video decoder to an increase in error rate at instant (or frame) $t . G$ is a constant that depends on several implementation issues, such as packetization, resynchronization, the encoded video sequence, etc. $\Gamma(t)$ is the squared error between the decoded video frame at instant $t$ and the previous one (both with no errors). Further details of the model are given in the Appendix.

\section{b) $N S V B R+E-E T$ and $N S V B R+I-E R$}

Similarly, assuming that the error at $t=0$ is successfully canceled at $t=T_{E-E T}$, from (A-6) we can show that the video distortion owing to residual channel errors achieved by NSVBR+E-ET is [42]

$$
D_{C H}(t)=P_{B, R} G \Gamma(t) \oplus \frac{\left[u(t)-u\left(t-T_{E_{-} E T}\right)\right]}{1+\theta t}
$$

with $P_{B, R}$ given by (12) (in general, note that $T_{E-E T}>T_{C V Q R}$ since E-ET takes place between endusers).

Finally, for NSVBR+I-ER we obtain

$$
D_{C H}(t)=P_{B, R} G \Gamma(t)
$$

which is derived from (A-6) with $T_{\max }=1$ video frame. 


\subsection{NUMERICAL RESULTS AND DISCUSSION}

We use the general simulation conditions described in subsection 3.4. The round trip delay is $N=6$ and the transmission rate is $R S 2$. The recovery time delays are $T_{C V Q R}=1 \mathrm{~s}$ and $T_{E-E T}=1.5 \mathrm{~s}$.

The average PSNRs for the video test sequences Mother\&Daughter and Foreman are presented in Figs. 10 and 11 , respectively. We present theoretical results derived from (18) with $D_{C H}(t)$ given by (21), (22), and (23) for CVQR, E-ET and I-ER, respectively. The average $D_{S C}(t)$ is evaluated using the distortion rate (DR) model [13].

Compared with an unprotected system, a significant gain can be observed. This gain depends on the residual block error rate and the amount of motion present in the sequence. Furthermore, it can be verified that NSVBR+CVQR outperforms NSVBR+E-ET in all the range of $P_{B}$. This is because

- E-ET takes place between end-users so the recovery time delay is larger than that in CVQR, and

- it does not consider packet losses between the last frame for which acknowledgment was received, and the current frame [40].

Comparisons with I-ER show that a residual average distortion exists in CVQR, which originates in the time delay required to stop error propagation (i.e., $T_{C V Q R}>1$ video frame).

Figure 12 shows video frames obtained with NSVBR and NSVBR+CVQR for $P_{B}=0.041$. Note that the residual degradation in the decoded video for NSVBR+CVQR is negligible in all cases.

It is important to realize that the improvements achieved by NSVBR+CVQR are obtained with low additional complexity and without modifying $\mathrm{H} .263$ codecs. In addition, note that the benefits of NSVBR can be enhanced using different retransmission strategies such as hybrid schemes [26]-[29]. This way, it is possible to improve not only video quality (since packet loss is reduced), but also system capacity. This is because the compression efficiency of the correction frames (CFs) improves, so the bandwidth requirements to transmit them are reduced.

\section{CONCLUDING REMARKS}

Based on novel error correction and error resilience techniques, this work has introduced a new architecture for robust interactive video transmission in MC-CDMA cellular networks. Our results have shown that the new protection method significantly improves the reliability of low bit rate video transmission over CDMA cellular systems. In addition, we showed that our approach outperforms previous protection schemes with small additional complexity. Furthermore, this paper has introduced a new model to evaluate performance of wireless video transmission. Although we have considered video transmission over slow fading channels, the architecture proposed here can be efficiently used in other environments ${ }^{4}$. Finally, it is important to realize the significance of the problem addressed in this work for practical situations: as an example, suffice it to consider the possibility of video transmission over mixed wired and wireless networks such as an H.324 based wired connection with an IS-95B mobile user.

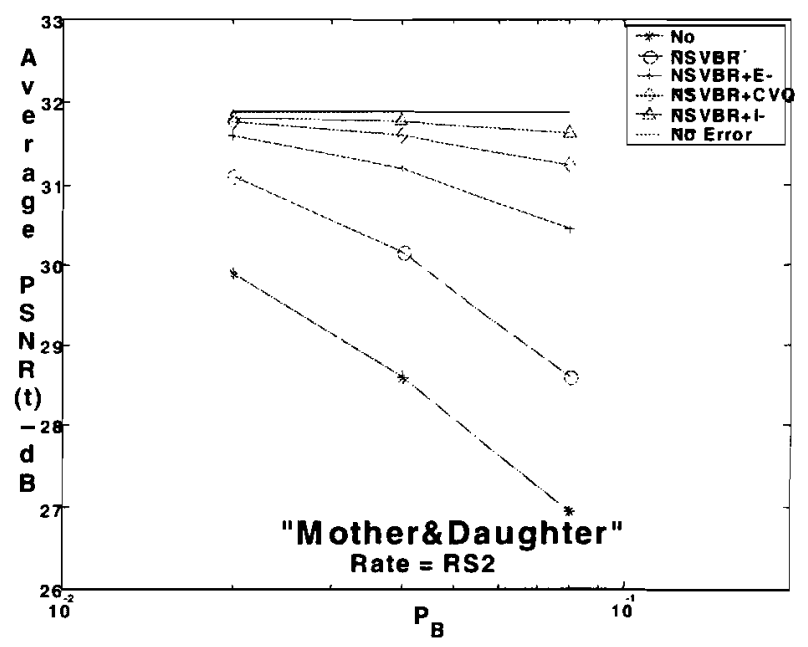

Figure 10. Average PSNR of sequence Mother\&Daughter.

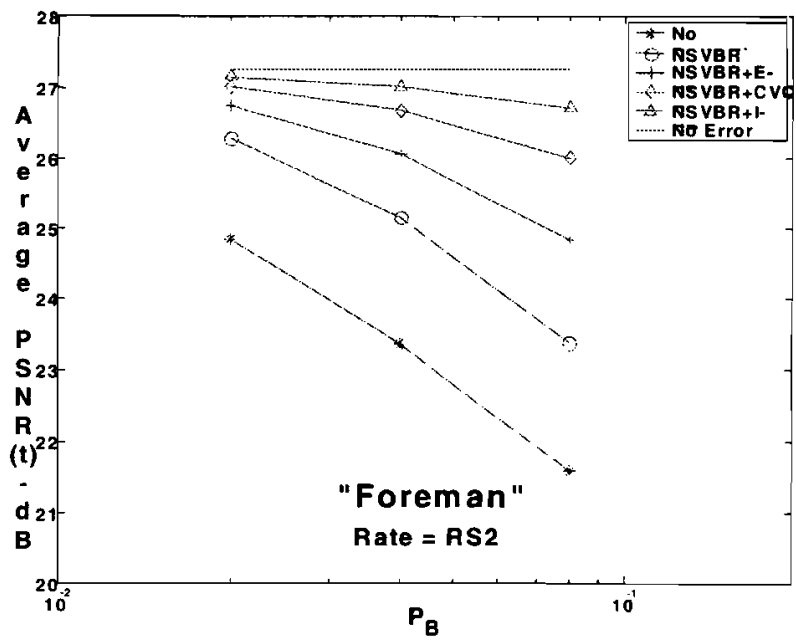

Figure 11. Average PSNR of sequence Foreman.

\footnotetext{
+ For example, the use of a selective variable bandwidth retransmission scheme (SVBR) should be considered for transmission in fast fading environments, or when closed-loop power control is used.
} 

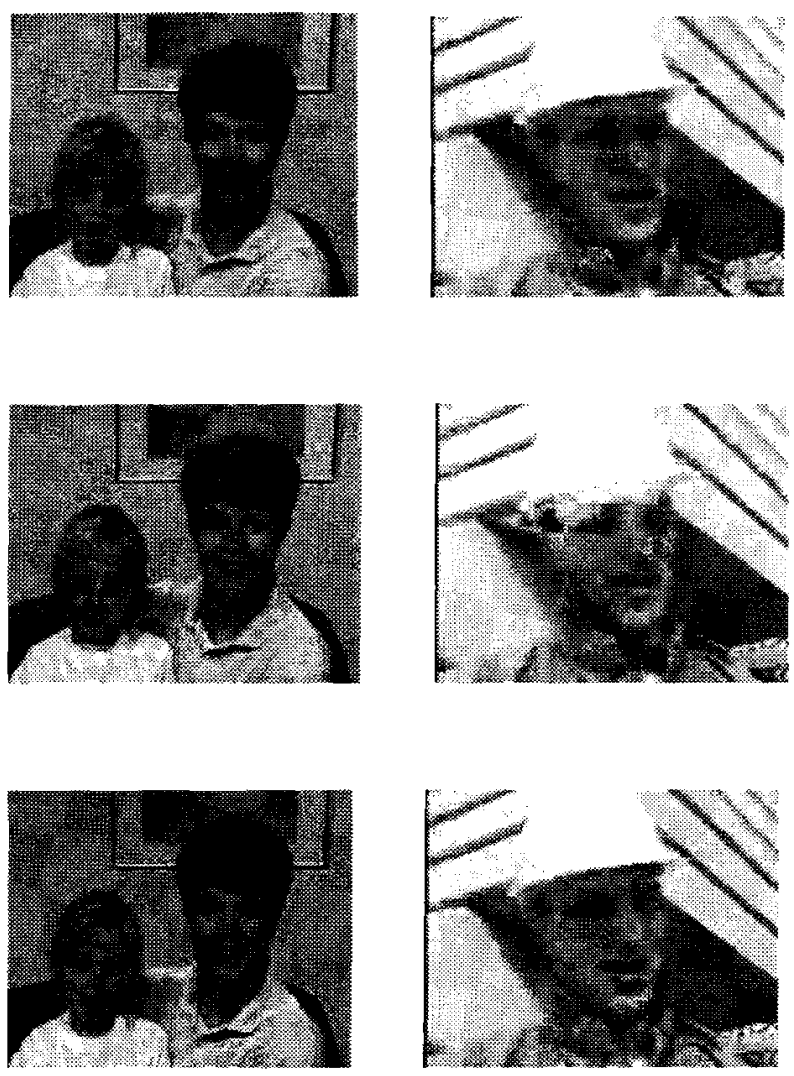

Figure 12. Frame 45 of sequence Mother \& Daughter (left). Frame 38 of sequence Foreman (right). Top: original (no errors). Middle: NSVBR. Bottom: NSVBR+CVQR.

\section{ACKNOWLEDGMENT}

The authors would like to thank Diego Crivelli for his help on preparing the final manuscript.

\section{APPENDIX}

This Appendix introduces a new model to estimate $D_{C H}(t)$ in transmission of $P$-frames over lossy channels. Towards this end, we present first the model proposed in [13]. It is assumed that an error signal is introduced at $t=0$ (i.e.. there is no error in the video frames or $D_{C H}(t)=0$ for $t<0$ ). The resulting error sequence is processed, before the video decoder, by the error concealment technique described in subsection 2.1. This introduces distortion for moving image regions, which propagates spatially and temporally until an intra-coded refresh is applied at $t=T_{\text {max }}$

Let $v(x, y, t)$ be the accumulated error signal for the video frame at instant $t$, which is the difference between the reconstructed frames at encoder and decoder. Let $u(x, y)$ be the residual error introduced at $t=0$ (i.e., $v(x, y, 0)=u(x, y))$. It has been shown in [13] that the variance of the propagated error signal at instant $t$ due to the error introduced at $t=0$ is given by

$$
\sigma_{v}^{2}(t)=P_{B} \sigma_{0}^{2} \underset{1+\theta t}{1}, \quad 0 \leq t<T_{\max } .
$$

$P_{B}$ is the block error probability and depends on the transmission system and channel characteristics (e.g., channel code, number of RAKE fingers, etc.). Parameter $\sigma_{0}^{2}$ represents the sensitivity of the video decoder to an increase in error rate, and its value depends on several implementation issues, such as packetization. resynchronization, and error concealment, as well as the encoded video sequence. It can be considered as a constant that does not depend on the other model parameters. Note that $P_{B} \sigma_{0}^{2}$ is the error variance introduced in the video sequence at $t=0$. The leakage $\theta$ describes the efficiency of explicit and/or implicit loop filtering to remove the introduced error. The range of typical values is given by $0<\theta<1$.

The model given by (A-1) has been used in [13] 10 analyze video transmission over lossy channels. In particular, time averaged video distortion has been considered in [13]. In this work we are interested not only in the time averaged video distortion owing to transmission errors, but also in the temporal progression of the distortion $D_{C H}(t)$. This model is useful because it allows, for example, the analytic evaluation of the performance of numerous error resilience techniques, as we presented in subsection 5.1. Assuming that the error signals $u(x, y)$ are uncorrelated frame to frame, and the sensitivity of the video decoder to an increase in error rate is constant (i.e., the error process is stationary), the total accumulated distortion is [13]

$$
D_{C H}(t)=\sum_{\tau=0}^{t} \sigma_{v}^{2}(\tau)=P_{B} \sigma_{0}^{2} \sum_{\tau=0}^{t} \frac{1}{1+\theta \tau}, \quad 0 \leq t<T_{\max } \text {. }
$$

Parameters $\theta$ and $\sigma_{0}^{2}$ can be determined by matching the model to measurement points for a complete video sequence.

The accuracy of this model degrades for video sequences with rapid changes of the motion patterns, such as an almost static video scene with an embedded short sequence of heavy motion (in this case, the effect of lost packets is not constant so $\sigma_{0}^{2}$ varies with $t$ ) [42], [43]. Since this problem is more critical in video transmission at low data rate (which is of interest for MC-CDMA cellular networks), it can be inferred that new approaches, such as the one introduced in this work, must be derived.

To improve the accuracy of (A-2), we propose to include in it the temporal variations of $\sigma_{0}^{25}$. Let $\sigma_{0}^{2}(t)$ be the sensitivity of the video decoder to an increase in error rate at instant $t$. Assuming that the error signals are uncorrelated frame to frame, the total accumulated distortion is

$$
\begin{gathered}
D_{C H}(t)=P_{B}\left[\sigma_{0}^{2}(0) \frac{1}{1+\theta t}+\sigma_{0}^{2}(1) \frac{1}{1+\theta(t-1)}+\ldots\right. \\
\left.\ldots+\sigma_{0}^{2}(t-1) \frac{1}{1+\theta}+\sigma_{0}^{2}(t)\right]
\end{gathered}
$$

or equivalently,

\footnotetext{
${ }^{5}$ Although parameter $\theta$ also varies with $t$, it has been verified that the effect of this variation over $D_{C H}(t)$ is negligible, therefore it can be considered as a constant [43].
} 


$$
D_{C H}(t)=P_{B} \sigma_{0}^{2}(t) \oplus \underset{1+\theta t}{1}, \quad 0 \leq t<T_{\max },
$$

where $\oplus$ denotes convolution. $\sigma_{0}^{2}(t)$ depends on factors such as packetization, resynchronization, nature of channel errors and error concealment, as well as the encoded video sequence. In particular, because of the error concealment technique used in this work, $\sigma_{0}^{2}(t)$ depends strongly on the difference between the video frames decoded at instant $t$ and the previous one. Therefore, if the change of the motion pattern between two consecutive video frames is large, the sensitivity of the video decoder to an increase in error rate will be high. On the other hand, video sequences with low mobility will have a low sensitivity to channel errors. Based on these observations, we propose modeling $\sigma_{0}^{2}(t)$ by

$$
\sigma_{0}^{2}(t)=G \Gamma(t)
$$

where $\Gamma(t)$ represents the squared error between the decoded video frame at $t$ and the previous one (both with no errors), while the constant $G$ takes into account the rest of the effects.

Finally, the distortion $D_{C H}(t)$ is

$$
D_{C H}(t)=P_{B} G \Gamma(t) \oplus \frac{1}{1+\theta t}, \quad 0 \leq t<T_{\max } .
$$

Similar to [13], for a given packetization and video sequence, parameters $\theta$ and $G$ can be estimated by fitting (A-6) to measurement points obtained from simulations (see [42] for more details). The improvements of accuracy of our approach over (A-2) have been demonstrated through comparisons with experimental results in [42].

\section{REFERENCES}

[1] TIA/EIA-95B. "Mobile station-base station compatibility standard for dual-mode wideband spread spectrum cellular system". July 1997.

[2] Chih-Lin I and R. D. Gitlin, "Multicode CDMA wireless personal communications networks," Proc. of IEEE ICC, vol. 2, pp. 1060-1064, 1995.

[3] V. K. Garg, IS-95 CDMA and cdma2000 - Cellular/PCS Systems Implementation. Prentice-Hall Inc, 2000.

[4] S. Kumar and S. Nanda, "High data-rate packet communications for cellular networks using CDMA: algorithms and performance," IEEE Journal on Select. Areas in Comm., vol. 3, pp. 472-492, March 1999.

[5] S. Lee, H. W. Lee, and D. K. Sung, "Capacities of single code and multicode DS-CDMA systems accommodating multiclass services," IEEE Trans. on Vehicular Tech., vol. 48, pp. 376-384, March 1999.

[6] D. Ayyagari and A. Ephremides, "Cellular multicode CDMA capacity for integrated (voice and data) services." IEEE Journal on Select. Areas in Comm, vol. 17, pp. 928-938, May 1999.

[7] ITU, Tel. Stand. Sector ITU, "Video coding for narrow telecommunication channels at< $64 \mathrm{kbit} / \mathrm{s}$," ITU-T Recom. H.263, 1996
[8] ITU, Tel. Stand. Sector ITU, "Video coding for narrow telecommunication channels at< $64 \mathrm{kbit} / \mathrm{s}$," Draft ITU-T Recom. H.263 Version 2, 1997.

[9] G. Côté, B. Erol, M. Gallant, and F. Kossentini, "H.263+: Video coding at low bit rates," IEEE Trans. Circuits Syst. Video Techn., vol. 18, pp. 849-866. November 1998.

[10] P. Bahl, "Supporting digital video in a managed wireless network," IEEE Comm. Magazine, pp. 94-102, June 1998.

[11] Y. Wang and Q-F Zhu. "Error control and concealment for video communication: a review," Proc. of IEEE, vol. 86, pp. 974-997, May 1998.

[12] M. Liu, W-C Feng, U. Mitra, and M. T. Liu, "Managing code assignment in multicode CDMA wireless networks for the delivery of H.263 video streams," Proc. of Int. Conf. on Computer Networks and Mobile Computing, pp. 473-478, 2001.

[13] K. Stuhlmüller, N. Färber, M. Link, and B. Girod, "Analysis of video transmission over lossy channels," IEEE Journal on Select. Areas in Comm., vol. 18, pp. 1012-1032, June 2000.

[14] M. R. Hueda, "A novel architecture for robust video transmission in MC-CDMA cellular networks over slow fading channels," Proc. of IEEE Globecom. vol. 2, pp. 17431748, Taipei, November 2002.

[15] N. Färber, B. Girod, and J. Villasenor, "Extensions of ITU-T recommendation H.324 for error-resilient video transmission," IEEE Comm. Magazine, pp. 120-128, June 1998.

[16] N. Chan and P. Mathiopoulos, "Efficient video transmission over correlated Nakagami fading channels for IS-95 CDMA systems," IEEE Journal on Select. Areas in Comm., vol. 18. pp.996-1010, June 2000.

[17] M. R. Hueda, C. Rodríguez, and C. Marqués, "H.263-based wireless video transmission in multicode CDMA systems," Proc. of 2001 IEEE VTC, pp. 433-437, Atlantic City, October 2001.

[18] G. de los Reyes, A. Reibman, S-F Chang, and J. Chuang, "Error resilient transcoding for video over wireless channels," IEEE Journal on Select. Areas in Comm., vol. 18, pp. 10631074. June 2000.

[19] R. Anand, C. Poldilchuk, and H. Lou, "Progressive video transmission over a wired-to-wireless network," Proc. of IEEE VTC, vol. 3, pp. 2424-2428, Tokio. 2000.

[20] H. Gharavi and S. Alamouti, "Multipriority video transmission for third-generation wireless communication systems," Proc. of IEEE, vol. 87, pp. 1751-1763, October 1999.

[21] M. Khansari, A. Jalali, E. Dubois, and P. Mermelstein, "Low bit-rate video transmission over fading channels for wireless microcellular systems," IEEE Trans. Circuits Syst. Video Techn, vol. 6, pp. 1-11, Feb. 1996.

[22] B. Girod and N. Färber, "Feedback-based error control for mobile video transmission," Proc. of IEEE, vol.87, pp. 872881, October 1999.

[23] E. Steinbach, N. Färber, and B. Girod, "Standard compatible extension of $\mathbf{H} .263$ for robust video transmission in mobile environments," IEEE Trans. Circuits Syst. Video Techn., vol. 7, pp. 872-881, December 1997.

[24] A. S. Tosun and W. Feng. "On improving quality of video for H.263 over wireless CDMA networks," Proc. of IEEE WCNC, pp. 1421-1426, Chicago, 2000.

[25] C. Hsu, A. Ortega, and M. Khansari, "Rate control for robust video transmission over burst-error wireless channels," IEEE Journal on Select. Areas in Comm., vol. 17, pp. 756-773, May 1999.

[26] S. Wang, H. Zheng, and J. Copeland, "A QoS enhanced hybrid SR-ARQ for mobile video communications," Proc. of IEEE ICC, pp. 526-530, New Orleans, 2000.

[27] H. Liu and M. El Zarki, "Performance of H.263 video transmission over wireless channels using hybrid ARQ," 
IEEE Journal on Select. Areas in Comm., vol. 15, pp. 17751786, December 1997.

[28] Q. Zhang and S. Kassam, "Hybrid ARQ with selective combining for fading channels," IEEE Journal on Select. Areas in Comm., vol. 17. pp. 867-879. May 1999.

[29] D. Qiao and K. Shin, "A two-step adaptive error recovery scheme for video transmission over wireless networks." Proc. of IEEE Infocom, vol. 3, pp. 1698-1704, 2000.

[30] B. Deep and W. Feng, "Adaptive code allocation in multicode-CDMA for transmitting H.263 video," Proc. of IEEE WCNC. pp. 1003-1007, New Orleans, September 1999.

[31] P. R. Chang, C. F. Lin, and M. J. Wu, "Design of multicode CDMA systems for 3-D stereoscopic video over wireless ATM networks," IEEE Trans. on Vehicular Tech., vol. 49, pp. 334-356, March 2000.

[32] F. Fitzek, R. Morich, and A. Wolisz. "Comparison of multicode link-layer transmission strategies in 3Gwireless CDMA", IEEE Comm. Magazine, pp. 58-64, October 2000.

[33] D. N. Knisely, Q. Li, and N. S. Ramesh, "cdma2000: A thirdgeneration radio transmission technology," Bell Labs Technical Journal, vol. 3, number 3, pp. 63-78, JulySeptember 1998.

[34] M. R. Hueda, C. E. Rodríguez, and C. Marqués, "Enhancedperformance video transmission in multicode CDMA wireless systems using a feedback error control scheme," Proc. of IEEE Globecom, San Antonio, vol. 1, pp. 619-626. December 2001.

[35] M. R. Hueda, "A Markov-based model for performance evaluation in multimedia CDMA wireless transmission," Proc. of IEEE VTC. pp. 668-673, Boston. September 2000

[36] J. Proakis Digital Communications. $3^{\text {rd }}$ ed. New York. McGraw-Hill, 1995.

[37] M. R. Hueda, G. Briones, and C. Rodríguez, "MMSECRAKE receivers with resolution reduction of the diversity branches: analysis, simulation and applications," IEEE Trans. on Communications, vol. 49, pp. 1073-1081, June 2001.

[38] W. C. Jakes Jr. Microwave Mobile Communications. New York, Wiley, 1974.

[39] ITU Telecom. Standardization Sector of ITU, "Video codec test model near-term, Ver. 8 (TMN8), Release 0," H.268 Ad Hoc Group, June 1997.

[40] R. Zhang, S. Regunathan, and K. Rose, "Video coding with optimal inter/intra switching for packet loss resilience," IEEE Journal on Select. Areas in Comm., vol. 18, pp. 966-976, June 2000.

[4I] I. Rhee and S. R. Joshi, "Error Recovery for Interactive Video Transmission over the Internet," IEEE Journal on Select. Areas in Comm., vol. 18, pp. 1033-1049, June 2000.

[42] M. R. Hueda, "Modeling low bit rate video transmission in CDMA wireless systems," Proc. of IEEE WPMC, vol. 3, pp. 1113-1117, Honolulu, Hawaii, October 2002.

[43] K. Stuhlmüller, N. Färber, M. Link, and B. Girod, "Adaptive optimal intra update for lossy video transmission," Proc. of $V C I P$, pp. 286-295, Perth, Australia, June 2000.

[44]F. Fitzek and M. Reisslein , "A prefetching protocol for continuous media streaming in wireless environments", IEEE Journal on Select. Areas in Comm., vol. 19, pp. 2015-2028. October 2001.
Mario R. Hueda was born in Jujuy, Argentina, in 1967. He received the B.S. in Electrical Electronic Engineering and the Ph.D degree from the National University of Cordoba, Argentina, in 1994 and 2002, respectively. From March 1994 to 1996 he received a fellowship from CONICOR (Scientific and Technological Research Council of Cordoba) to develop advanced voiceband modems. During the summer of 1996 he was a visiting scholar with Lucent Technologies - Bell Labs in Murray Hill, USA, where he worked on CDMA receivers. Since 1997 he has been with the Digital Communications Laboratory at the Department of Electronic Engineering of the National University of Cordoba. His research interests include wireless video transmission, performance of CDMA systems and digital communications.

Carmen E. Rodríguez received the Electrical and Electronic Engineer degree from the National University of Cordoba, Argentina, in 1984. From 1984 to 1991 she was with the Electronic Instrumentation Laboratory at the Mathematics and Physics Department of the National University of Cordoba as a Research Associate. In 1992 she became Associate Professor at the Electronic Engineering Department. In 1996 she founded the Digital Communications Research Laboratory, of which she is currently Director. Professor Rodríguez is also Vice-President of CIEC (Institute of Electrical, Electronics, Mechanical and Chemical Engineering of Cordoba, Argentina), President of the Argentinean Society of Engineering, and Member of the Mercosur Technological Area Commite. 